\title{
A new paradigm for delivering personalised care: integrating genetics with surgical interventions in BEST1 mutations
}

\author{
Sancy Low $\mathbb{D}^{1,2,3} \cdot$ Ryian Mohamed $\mathbb{1}^{4}$ - Alice Davidson ${ }^{2,5} \cdot$ Maria Papadopoulos $^{1} \cdot$ Piergiacomo Grassi $^{1,3}$. \\ Andrew R. Webster $\mathbb{D}^{1,2,5}$. Graeme C. Black $\mathbb{1}^{6,7}$. Paul J. Foster $\mathbb{D}^{1,2,5}$ - David F. Garway-Heath $\mathbb{D}^{1,2,5}$. \\ Philip A. Bloom ${ }^{8}$
}

Received: 30 December 2018 / Revised: 2 June 2019 / Accepted: 25 June 2019 / Published online: 27 August 2019

(c) The Author(s), under exclusive licence to The Royal College of Ophthalmologists 2019

\begin{abstract}
Background The availability and reduced cost of genotyping has improved gene susceptibility testing and our scientific understanding of disease pathophysiology. Whilst several personalised translational models exist within medical frameworks, genetic-based surgical therapy is a translational application not widely used in surgical specialties.

Method We present a clinical series of five patients with genetically confirmed bestrophinopathy and malignant glaucoma (MG). Patients were followed up for 12 months or more after receiving surgical intervention to manage refractory intraocular pressure (IOP) resistant to medical treatment.

Findings Patients with BEST1 gene mutations are at higher risk of MG after filtration surgery. A multi-disciplinary approach after four patients experienced poor outcomes concluded that traditional first-line glaucoma surgery was not sufficient to prevent visual loss. A fifth patient presenting with the identified at-risk phenotype underwent primary pars plana vitrectomy, with pars plana Baerveldt tube insertion, successfully preventing MG and had no glaucoma progression after 5 years.

Interpretation We provide proof-of-principle that genetic analysis can be used to inform the selection of surgical therapy to improve outcomes. In this case, a refinement of current surgical methods to avoid MG. Although challenges remain, personalised surgery has the potential to improve clinical outcomes beyond the scope of current surgical practice.
\end{abstract}

Supplementary information The online version of this article (https:// doi.org/10.1038/s41433-019-0553-5) contains supplementary material, which is available to authorized users.

Sancy Low

sancy.low@ucl.ac.uk

1 Moorfields Eye Hospital NHS Foundation Trust, London, UK

2 UCL Institute of Ophthalmology, London, UK

3 Department of Ophthalmology, Guys and St. Thomas' Hospital NHS Foundation Trust, London, UK

4 Department of Ophthalmology, Central Middlesex Hospital, London, UK

5 NIHR BRC for Ophthalmology at Moorfields Eye Hospital and UCL Institute of Ophthalmology, London, UK

6 Centre for Genomic Medicine, Institute of Human Development, Faculty of Medical and Human Sciences, University of Manchester, Manchester, UK

7 Central Manchester University Hospitals NHS Foundation Trust, Manchester, UK

8 Imperial College NHS Foundation Trust, London, UK

\section{Introduction}

Personalised medicine (PM) is the translational goal at the core of current molecular medical research [1]. How to effectively, efficiently and economically integrate genetic information into the medical management of patients is the biggest challenge in frontline innovation strategies [2, 3]. Using genetic information to guide the selection of surgical candidates has now been reported in cancer therapy [4] and neurosurgery [5], with likely applications in more specialties.

Best disease (BD) was first described by Adams in 1883 and named by Best in 1905 [6]. It is an inherited, macular dystrophy characterised by vitelliform macular lesions (egg yolk appearance), a normal electroretiongram (ERG) and loss of the electooculogram (EOG) light-rise. The aetiology of $\mathrm{BD}$ was first linked to the BEST1 (VMD2) gene in 1998 [7]. BD is classically an autosomal dominant disorder (ADB). In 2008, a more devastating and underdiagnosed form autosomal recessive bestrophinopathy (ARB) was discovered, with a previously unknown risk of angle- 
closure glaucoma, which was present in at least $50 \%$ of patients with ARB [8]. We present a translational disease paradigm where genetically informed personalised surgical intervention can directly improve clinical outcomes, thereby coining the term "surgico-genetics".

At two tertiary referral centres for glaucoma, we observed a series of three patients with ARB (cases 1, 2 and 3 ) and one with ADB (case 4, the only autosomal dominant case with angle-closure glaucoma reported in the literature at the time of this report), who developed a sight threatening complication after first-line glaucoma surgery, known as malignant glaucoma (MG).

MG occurs when aqueous humour, fluid in the anterior chamber (AC) (between cornea and iris) is diverted into the posterior chamber (between iris, zonule fibres and lens) causing the intraocular pressure (IOP) to become uncontrollably high. Cases 1-4 had poor outcomes following initial surgery (cataract removal in patient 4 , and trabeculectomy in patients 1-3) and developed MG. Additional surgical management including cycloablation (laser destruction) of the ciliary body, further glaucoma drainage implant surgery, and long-term topical and oral medications became necessary. The full clinical details of cases 1-5 are presented below, and key clinical signs and relevant imaging for cases 1-5 are highlighted in the supplementary PowerPoint presentation.

\section{Methods}

\section{Genome sequencing and assembly}

Bidirectional Sanger sequencing of BEST1 in affected individuals was performed at the National Genetics Reference Library in Manchester, UK.

\section{Case reports}

\section{Case 1}

A 44-year-old Caucasian female previously diagnosed with bilateral autosomal recessive bestrophinopathy (ARB) presented with bilaterally closed angles, right glaucomatous optic neuropathy, IOPs being $34 \mathrm{mmHg} \mathrm{RE}$ and $26 \mathrm{mmHg}$ left eye (LE), best corrected visual acuity (BCVA) was 6/60 right eye (RE) and 6/36 LE. She underwent bilateral YAG laser iridotomies followed by right mitomycin augmented trabeculectomy. One week postoperatively, the patient presented with right severe ocular pain, shallow right $\mathrm{AC}$ and right IOP $47 \mathrm{mmHg}$, right $\mathrm{MG}$ was diagnosed and she underwent right pars plana vitrectomy (PPV) with subsequent AC reformation. Six months postoperatively, right BCVA had deteriorated to HM due to right cystoid macular oedema despite being on long-term oral acetazolamide $500 \mathrm{mg}$ bd, her IOPs were $11 \mathrm{mmHg}$ RE (on cyclopentolate $1 \%$ nocte) and $16 \mathrm{mmHg} \mathrm{LE}$ (on timolol $0.25 \%$ bd, latanoprost $0.005 \%$ nocte) with significant cupping of the right optic disc, examination showed bilateral juxtapapillary drusen. Molecular genetic analysis revealed two heterozygous variants: c. $102 \mathrm{C}>\mathrm{T}$ p.Gly34Gly and c.572T $>\mathrm{C}$ p. Leu191Pro at the BEST1 gene.

\section{Case 2}

A 41-year-old Caucasian male phakic with bilateral advanced primary angle-closure glaucoma (PACG) and bilateral ARB, exfoliation syndrome on the AC lens surface, and optic discs drusen presented with bilateral ocular pain and blurred vision for $12 \mathrm{~h}$, having had bilateral augmented trabeculectomies. BCVA was 6/60 RE and 3/60 LE, examination revealed shallow ACs (right $>$ left), flat hyperaemic blebs, IOPs measuring 43 and $25 \mathrm{mmHg}$, axial lengths (ALs) measuring 21.45 and $21.32 \mathrm{~mm}$, and $\mathrm{AC}$ depths (ACDs) measuring $2.05 \mathrm{~mm}$ and $2.36 \mathrm{~mm} \mathrm{RE}$ and LE, respectively. Bilateral MG was diagnosed (Fig. 1) and both eyes were firstly commenced on topical atropine $1 \%$ tds, and then underwent cyclodiode laser ciliary body ablation (CLCBA) 1 week and 9 months later RE and LE, respectively. Eight weeks after left CLCBA, IOPs measured $22 \mathrm{mmHg} \mathrm{RE}$ and $9 \mathrm{mmHg} \mathrm{LE}$ with partial recovery of BCVA in the RE to 6/24. Molecular genetic analysis revealed a mutation c.602 $\mathrm{T}>\mathrm{C}$ p (Ile201Thr) in exon 5 of the BEST1 gene. MG recurred after 4 months of CLCBA, but he also developed an allergy to atropine eye drops used for the treatment of MG.

\section{Case 3}

A 24-year-old student bilaterally phakic with bilateral ARB and bilateral PACG and left filtering bleb from previous augmented trabeculectomy presented for progressive blurred central vision in her RE over last 6 months. On examination, BCVA was 6/24 in RE and CF in LE, IOPs were $46 \mathrm{mmHg}$ and $16 \mathrm{mmHg}$, respectively, examination showed left MG (-6.75 spherical equivalent of the myopic shift in the $\mathrm{LE}+$ left $\mathrm{ACD}<2 \mathrm{~mm}$ ) and bilateral peripheral anterior synechiae. The patient initially underwent right iridotomy and iridoplasty, and then right clear lens extraction + IOL 2 weeks later, with subsequent right aqueous misdirection 1 week postoperatively, right IOP having progressively increased from 14 to $35 \mathrm{mmHg}$ and right AC being shallow. She was initially commenced on $\mathrm{g}$. atropine $1 \%$ to both eyes, and then underwent right CLCBA and left clear lens extraction + IOL with subsequent left bleb failure, and then underwent left CLCBA (Fig. 2). Four months postoperatively, BCVA was 6/36 in both eyes, her IOPs being 


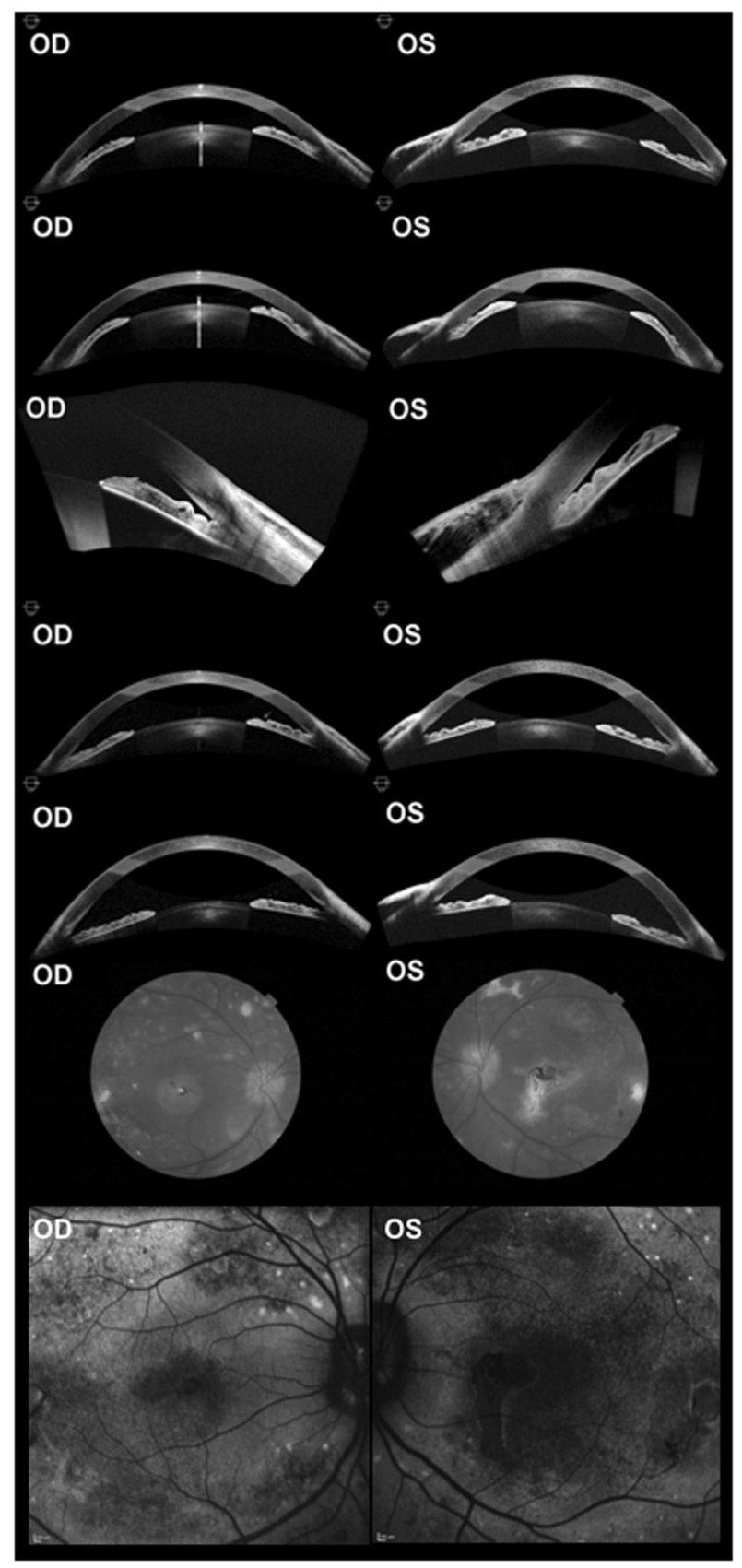

Fig. 1 Anterior segment-OCT images of Case 2. First row: anterior segment-OCT images the day after tapering down g. atropine $1 \%$ from tds to od in both eyes. Second row: anterior segment-OCT images during the second aqueous misdirection episode in both eyes, during treatment with $\mathrm{g}$. atropine $1 \%$ od. Third row: anterior segment-OCT images of anterior chamber angles, showing reduced angle opening distance 500 (AOD 500), and the marked shallowing of the anterior chamber during aqueous misdirection in both eyes. Fourth row: anterior segment-OCT images during initial resolution of aqueous misdirection in the left eye, 3 days after left CLCBA. Fifth row: anterior segment-OCT images 8 weeks after left CLCBA. Aqueous misdirection has now resolved in both eyes. Sixth and seventh row: colour photographs and fundus autofluorescence with bilateral advanced PACG, showing bilateral ARB, bilateral optic discs drusen and macular perifoveal lesions typical of ARB
16 and $26 \mathrm{mmHg}$ respectively on dorzolamide $2 \% /$ timolol $0.5 \%$ bd and latanoprost $0.005 \%$ nocte to both eyes with left fully cupped disc. Molecular genetic analysis revealed the c. $454 \mathrm{C}>\mathrm{G}$ and the c. $481+1 \mathrm{G}>\mathrm{T}$ mutations at the BESTI gene.

\section{Case 4}

A 42-year-old Caucasian female bilaterally phakic, with bilateral ADB, bilateral ACG and previous LE multiple filtering surgeries which led to corneal endothelial damage and opacities presented for a second opinion about her RE. Her right IOP was $43 \mathrm{mmHg}$ on four topical lowering-IOP drugs, she initially underwent right clear lens extraction + IOL and developed aqueous misdirection 1 week postoperatively. Despite a deepening of her right AC with topical atropine $1 \%$, right IOP remained $40 \mathrm{mmHg}$ requiring right CLCBA, and right IOP normalised to $10 \mathrm{mmHg}$ on no topical/systemic anti-glaucoma treatment 9 months postCLCBA. EOG showed neither dark troughs nor light-rise in both eyes, confirming the ADB diagnosis. Molecular genetic analysis the coding region (exon 8), c.914 T>C, p.Phe305Ser.

\section{Case 5}

A 23-year-old lady with deteriorating vision for 4 years, BCVA 6/60 RE, hand motion LE, was found to have elevated IOP of $38 \mathrm{mmHg} R E$ and $48 \mathrm{mmHg} \mathrm{LE}$, and bilateral vitelliform lesions at the macula. Her optic disc and anterior segment examination showed end stage angle-closure glaucoma, with disc cupping $>0.9$ in both eyes. Due to her young age and significant risk of complete visual loss, a multi-disciplinary approach was taken while temporising surgery with cycloablation was performed, and genetic testing to confirm her BEST1 disease status.

Her IOPs remained uncontrolled with maximal medical therapy and acetazolamide, so definitive surgery with primary PPV (complete vitreous gel removal) and a glaucoma drainage implant (Baerveldt tube) directly through the pars plana into the posterior chamber was performed sequentially in both eyes - see also Supplementary material.

After 2 months, her IOPs normalised to $12 \mathrm{mmHg}$ RE and $17 \mathrm{mmHg}$ LE. After 5 years, her IOPs were $16 \mathrm{mmHg}$ in both eyes with no medications required in both eyes. There was reversal of her optic disc cupping to 0.7 in the RE, and stable at 0.9 in her LE. She achieved the best IOP and vision outcome of any patient studied, avoiding the complication of MG throughout her 5-year treatment course. This was a direct result of selecting primary vitreous removal surgery, combined with a pars plana drainage implant, when we recognised her genetic risk of refractory 
Fig. 2 Colour photographs, visual fields and anterior segment imaging of patient 3 . The right eye is shown in $\mathbf{a}-\mathbf{d}$, left eye in e-g. Significant iridotrabecular contact was seen in the right eye on AS-OCT (a), and upon instillation of $\mathrm{g}$. pilocarpine $2 \%$ drops, the angle remained closed (b). One week after right clear lens extraction (c), the angles were still closed on gonioscopy and AS-10.1038/ s41433-019-0553-5 OCT. The anterior chamber (AC) continued to shallow over 5 weeks, requiring g. atropine drops to deepen the AC, and treat any subclinical aqueous misdirection. d A good response to $\mathrm{g}$. atropine, the IOP was lowered and more open $\mathrm{AC}$ angles were seen. The left eye underwent trabeculectomy before these images were taken $(\mathbf{e}, \mathbf{f})$. f A superior bleb is on the left side of this cross-sectional image, taken vertically. On high resolution AS-OCT, significant iridocorneal contact completely occluding the anterior chamber angle is seen in f. After clear lens extraction in the left eye, the AC shallowed initially, but deepened after cyclodiode ablation of the ciliary body (g). The IOP in the left eye remains high, despite deepening of the drainage angle and the patient subsequently had an anterior chamber Baerveldt tube inserted for IOP control
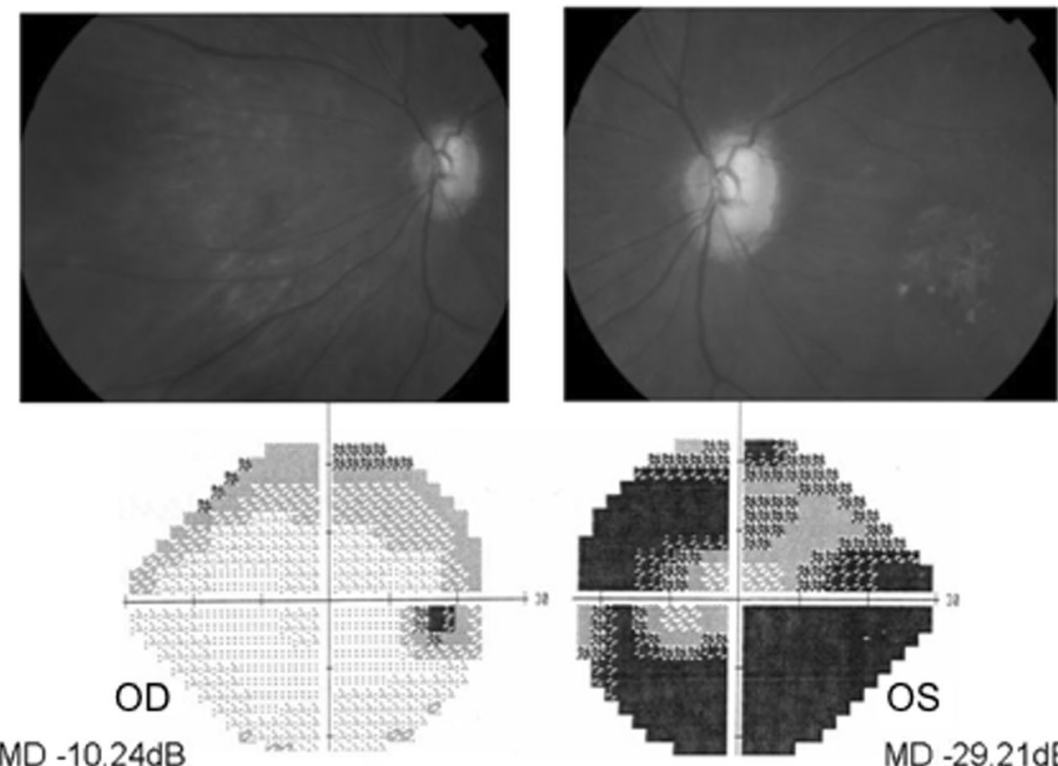

MD $-10.24 d B$

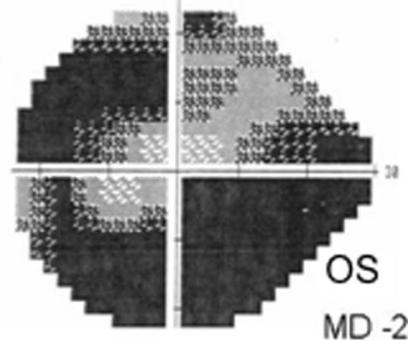

$-29.21 d B$
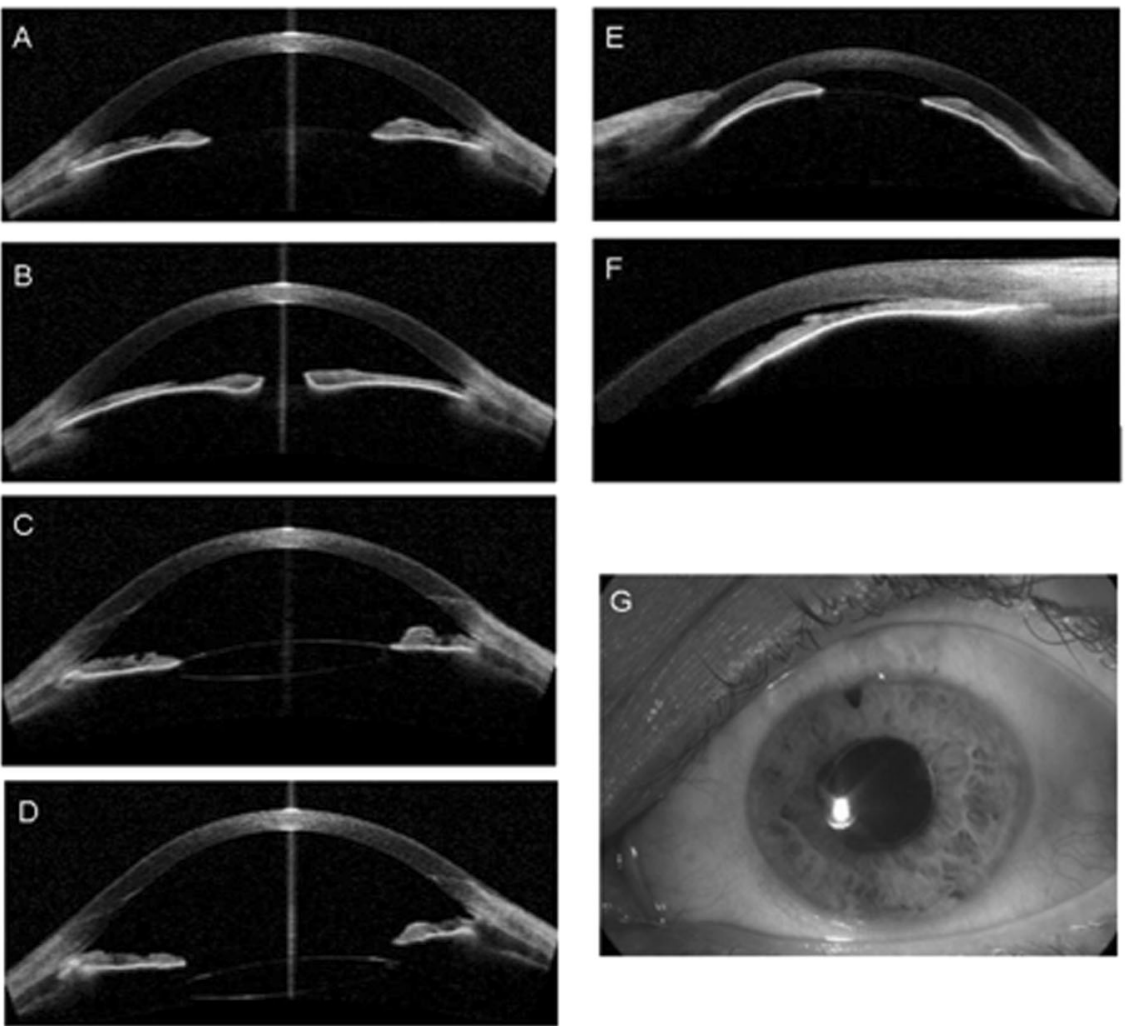

MG that was observed in the other patients who had firstline surgery not personalised to their underlying genetic condition (see Fig. 3).

\section{Discussion}

The BEST1 gene encodes bestrophin-1, a transmembrane protein located in the basolateral membrane of the retinal pigment epithelium (RPE) [9-12] The light-rise of the EOG is thought to result from chloride conductance across the basolateral membrane of the RPE [13, 14]. Exogenous expression of bestrophin-1 produces a chloride ion conductance, which suggests that bestrophin-1 may function as a calcium-sensitive chloride channel directly responsible for generating the light-rise of the EOG [15].

Half of all mutations described are found in the C-terminal region of bestrophin-1, which interacts with serine/threonine 


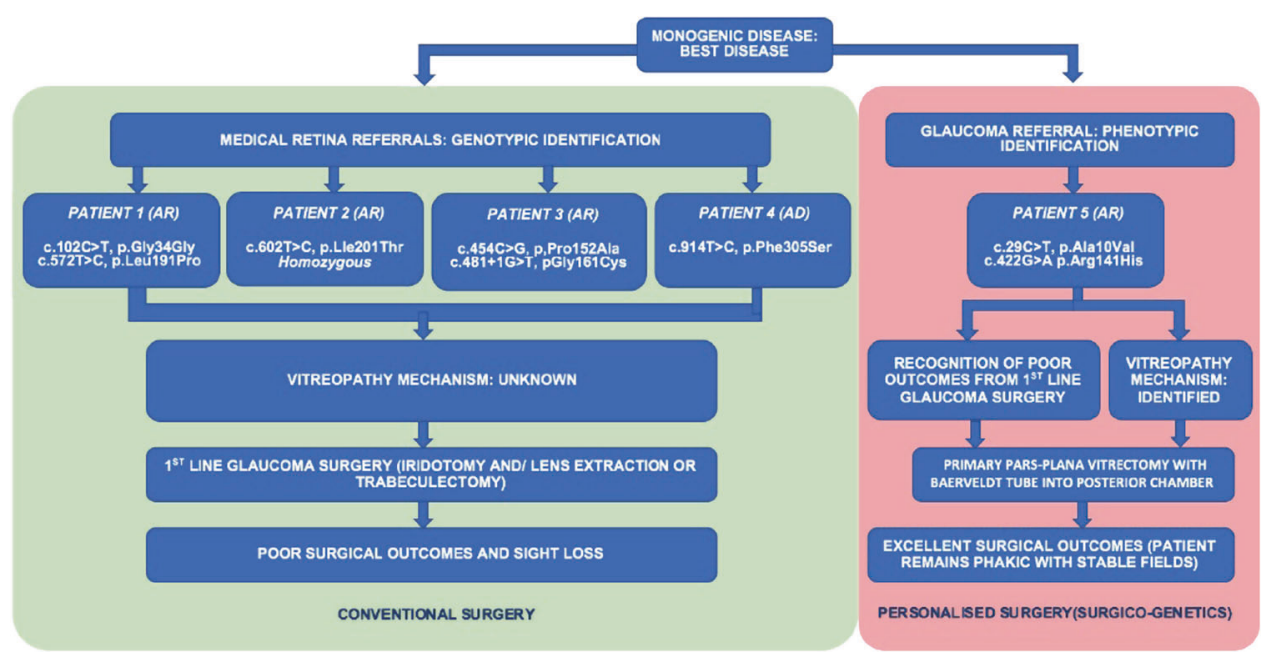

Fig. 3 The personalised surgical (surgico-genetic) model for managing angle-closure glaucoma secondary to BEST1 mutations. In sub-specialist clinics, the genetic aetiology of a condition may be recognised by a specialty group/physicians, while the surgical intervention is managed by a different specialty or surgical group. During joined-up care, careful recognition of any association between mutation status and adverse surgical outcomes provides a unique opportunity for further understanding of surgico-genetic interventions to personalise

protein phosphatase2A (PP2A), indicating that these residues may be important in the regulatory interaction between bestrophin-1 and phosphatase2A [16]. The protein $P P 2 A$ is important in the regulation of photoreceptors in the human retina, which might explain the ERG abnormalities noted in ARB. Phosphorylation and dephosphorylation of bestrophin1 may also act as the on/off light switch for the amplitude and timing of the EOG response [16].

Choroidal expansion [17] is the most likely mechanistic explanation for the phenotype of MG. Given that hyperopia of $>+3.0 \mathrm{DS}$ is a recognised feature of BD [18], shallow iridocorneal angles are expected. However, the consistent report of MG in ARB cases has now been discussed by Zhong et al. [19] and our series with $100 \%$ penetrance of MG after trabeculectomy, apart from case 5 in this report. This supports an inherent vitreopathy triggering MG when incisional surgery is performed (without removing the vitreous). A defective homoeostasis of the fluid exchange chloride pump is also highly probable [20].

The abnormal AL (small to nanophthalmic eyes in Bestrophin patients) and high myopia of ADVIRC patients are both under BEST1 genetic regulation [10, 21, 22]. We hypothesise that BEST1, which is found in the RPE, has closely regulated developmental functions with the vitreous and choroid, expressed as extremes in axial biometry $[23,24]$, and the development of MG, particularly when biallelic mutations (ARB) are present [21].

In case 5, personalised surgical treatment of vitrectomy disrupted the anterior hyaloid face, released any pockets of aqueous sequestered within the vitreous gel and re-established treatments. We provide an example here with BEST1 mutations. A known surgical intervention, primary pars plana vitrectomy and primary Baerveldt tube implantation in the posterior chamber, which addresses the vitreopathy, can become the treatment of choice when secondary angle-closure glaucoma is detected. This avoids the complications of malignant glaucoma that are highly frequent with treatments like lens extraction or trabeculectomy in the presence of BEST1 mutations. AR autosomal recessive, $\mathrm{AD}$ autosomal dominant

communication between anterior and posterior chambers. This avoided development of MG during her clinical course. Whilst the direct genotype-phenotype of this association cannot currently prove that the vitreous is involved in the disease, other authors have also clearly stated that patients with BEST1 mutations at risk of angle-closure glaucoma have to 'avoid filtration surgery/trabeculectomy'. Our paper presents an alternative surgical method with primary PPV and posterior chamber drainage tube implantation, which successfully bypassed the complication of MG, and remained functional after 5 years. We thereby highlight the translational implications of genetic information guiding surgical selection (surgico-genetics).

\section{Conclusion}

Actionability of genetic data is defined by Snyder et al. as the interpretation of molecular events to guide therapeutics and clinical care. The first paper describing genetic diagnosis changing the medical treatment of an individual patient was published in 2012 [23]. This report describes genetic analysis directly informing the selection of surgical options, not directly for the primary retinal disorder, but to manage and prevent devastating sight threatening complications from the associated glaucoma. As such, there is currently no recognition, regulatory framework or funding for the field which we call surgico-genetics.

Like clinical medicine, surgery too can be optimised by considering individual variables, for example, age, 
comorbidities, and psychological make-up. We believe surgico-genetics to be the next frontier in improving surgical outcomes for our patients. The logistics of achieving this within daily practice are possibly what has halted the genesis and development of this idea so far. By working closely with our research partners, the potential gains of personalised surgery may shift this trend.

Combined PPV surgery and pars plana Baerveldt tube implantation for refractory glaucoma has already been shown [24], and in this case applied when managing angleclosure glaucoma in a young patient with ARB, preserving her sight (Fig. 3) for more than 5 years to date, with no complications. Ophthalmology has an established record in the forefront of PM [25], and with more molecular diagnoses being made, we anticipate further progress in surgicogenetics both from our field, and other surgical specialties.

\section{Summary}

\section{What was known before}

- Best disease is a rare genetic disorder affecting the eye, mainly at the macula if autosomal dominant, and more severely affecting the macula and remaining fundus with autosomal recessive inheritance.

\section{What this study adds}

- Best patients have an additional risk of developing malignant glaucoma, which is particularly difficult to manage, and requires surgical intervention.

- Filtration surgery and trabeculectomy is not recommended in patients with bestrophinopathies.

- We demonstrate a solution describing a surgical technique based on an understanding of the molecular diagnosis.

- We provide proof-of-principle that genetic analysis can be used to inform the selection of surgical therapy.

\begin{abstract}
Acknowledgements The authors would like to acknowledge the valuable contribution of UCL Institute of Ophthalmology and Moorfields Eye Hospital NHS Foundation Trust. We would also like to individually thank Anthony Moore, Winifred Nolan, Faisal Ahmed and Laura Crawley. The authors would also like to acknowledge the contribution of both the glaucoma and vitreoretinal teams of the Western Eye Hospital in performing the primary pars plana vitrectomy and pars plana Baerveldt tube for Patient 5.
\end{abstract}

\section{Compliance with ethical standards}

Conflict of interest The authors declare that they have no conflict of interest.
Ethical approval Patients 1-4 were participants of research studies approved by the Moorfields Eye Hospital NHS Foundation Trust Ethics Committee. Patient 5 was a National Health Service patient and genotyping based on clinical need. All subjects received education and counselling and provided informed consent.

Publisher's note: Springer Nature remains neutral with regard to jurisdictional claims in published maps and institutional affiliations.

\section{References}

1. Personalized Medicine Coalition. Personalized medicine at FDA: progress report. Personalized Medicine Coalition. Washington; 2018.

2. Personalized Medicine Coalition. The personalized medicine report: opportunity, challenges, and the future. Personalized Medicine Coalition. Washington; 2018.

3. Ellen Graham. Improving outcomes through personalised medicine. NHS England. England; 2016.

4. Caso R, Beamer M, Lofthus A, Sosin M. Integrating surgery and genetic testing for the modern surgeon. Ann Transl Med. 2017;5:399

5. Stevelink R, Sanders MW, Tuinman MP, Brilstra EH, Koeleman BP, Jansen FE, Braun KP. Epilepsy surgery for patients with genetic refractory epilepsy: a systematic review. Epileptic Disord. 2018;20:99-115.

6. Beighton P, Beighton G. The Person Behind the Syndrome. London: Springer; 1997. BEST, Friedrich.

7. Petrukhin K, Koisti MJ, Bakall B, Li W, Xie G, Marknell T, et al. Identification of the gene responsible for best macular dystrophy. Nat Genet. 1998;19:241-47.

8. Burgess R, et al. Biallelic mutation of BEST1 causes a distinct retinopathy in humans. Am J Hum Genet. 2008;82:19-31.

9. Guziewicz KE, et al. Bestrophin gene mutations cause canine multifocal retinopathy: a novel animal model for best disease. Investig Ophthalmol Vis Sci. 2007;48:1959-67.

10. Marmorstein AD, et al. Bestrophin, the product of the best vitelliform macular dystrophy gene (VMD2), localizes to the basolateral plasma membrane of the retinal pigment epithelium. Proc Natl Acad Sci USA. 2000;97:12758-63.

11. Mullins RF, Kuehn MH, Faidley EA, Syed NA, Stone EM. Differential macular and peripheral expression of bestrophin in human eyes and its implication for best disease. Investig Ophthalmol Vis Sci. 2007; 48:3372-80.

12. Bakall B, et al. Expression and localization of bestrophin during normal mouse development. Investig Ophthalmol Vis Sci. 2003;44:3622-8.

13. Gallemore RP, Hughes BA, Miller SS. Retinal pigment epithelial transport mechanisms and their contributions to the electroretinogram. Prog Retin Eye Res. 1997;16:509-66.

14. Arden GB, Constable PA. The electro-oculogram. Prog Retin Eye Res. 2006;25:207-48.

15. Sun H, Tsunenari T, Yau KW, Nathans J. The vitelliform macular dystrophy protein defines a new family of chloride channels. Proc Natl Acad Sci USA. 2002;99:4008-13.

16. Low S, et al. Autosomal dominant best disease with an unusual electrooculographic light rise and risk of angle-closure glaucoma: a clinical and molecular genetic study. Mol Vis. 2011; 17:2272-82.

17. Sakai $\mathrm{H}$, et al. Uveal effusion in primary angle-closure glaucoma. Ophthalmology. 2005;112:413-9.

18. Wittstrom E, Ponjavic V, Bondeson ML, Andreasson S. Anterior segment abnormalities and angle-closure glaucoma in a family with a mutation in the BEST1 gene and best vitelliform macular dystrophy. Ophthalmic Genet. 2011;32:217-27. 
19. Zhong Y, et al. Flat anterior chamber after trabeculectomy in secondary angle-closure glaucoma with BEST1 gene mutation: case series. PLoS ONE. 2017;12:e0169395. 5

20. Qu Z, Hartzell HC. Bestrophin Cl- channels are highly permeable to HCO3. Am J Physiol Cell Physiol. 2008;294:C1371-7.

21. Reddy MA, Francis PJ, Berry V, et al. A clinical and molecular genetic study of a rare dominantly inherited syndrome (MRCS) comprising of microcornea, rod-cone dystrophy, cataract, and posterior staphyloma. Br J Ophthalmol. 2003;87:197-202.

22. Yardley J, Leroy BP, Hart-Holden N, et al. Mutations of VMD2 splicing regulators cause nanophthalmos and autosomal dominant vitreoretinochoroidopathy (ADVIRC). Investig Vis Sci. 2004;45:3683-9.

23. Worthey EA, et al. Making a definitive diagnosis: successful clinical application of whole exome sequencing in a child with intractable inflammatory bowel disease. Genet Med. 2011;13:255-62.

24. Luttrull $\mathrm{JK}$, et al. Initial experience with pneumatically stented baerveldt implant modified for pars plana insertion for complicated glaucoma. Ophthalmology. 2000;107:143-9. discussion 149-50

25. Black GCM, et al. Personalized ophthalmology. Clin Genet 2014;86:1-11. 